\title{
CORRECTION
}

\section{Correction to: Measuring physical performance in highly active older adults: associations with age and gender?}

\author{
Gro Gujord Tangen ${ }^{1,2,3}$ [D $\cdot$ Hilde Stendal Robinson ${ }^{1}$
}

Published online: 17 August 2019

๑) Springer Nature Switzerland AG 2019

\section{Correction to: Aging Clinical and Experimental Research https://doi.org/10.1007/s40520-019-01190-x}

In the published article, the Table 2 was published incorrectly. The correct Table 2 is given below.

The original article has been updated.

The original article can be found online at https://doi.org/10.1007/ s40520-019-01190-x.

Gro Gujord Tangen

g.g.tangen@medisin.uio.no

Hilde Stendal Robinson

h.s.robinson@medisin.uio.no

1 Department of Interdisciplinary Health Sciences, Institute of Health and Society, University of Oslo, P.O. Box 1089, Blindern, 0317 Oslo, Norway

2 Norwegian National Advisory Unit on Ageing and Health, Vestfold Hospital Trust, Tonsberg, Norway

3 Department of Geriatric Medicine, Oslo University Hospital, Oslo, Norway 
Table 2 Results on performance-based tests of physical function

\begin{tabular}{|c|c|c|c|c|c|}
\hline Test & All $(n=105)$ & $\operatorname{Men}(n=57)$ & Women $(n=48)$ & $B^{\mathrm{a}} / \mathrm{OR}^{\mathrm{b}}(95 \% \mathrm{CI})$ & $p$ \\
\hline \multicolumn{6}{|l|}{ General mobility } \\
\hline \multicolumn{6}{|l|}{ SPPB $(0-12)$} \\
\hline Median (IQR) & $12(2)$ & $12(1)$ & $12(2)$ & & \\
\hline Min-max & $4-12$ & $6-12$ & $4-12$ & & \\
\hline$>10$ points, $n(\%)$ & $79(75)$ & $44(77)$ & $35(73)$ & $1.8(0.7,4.9)^{\mathrm{a}}$ & $0.227^{\mathrm{c}}$ \\
\hline Gait speed, m/s, mean (SD) & $1.14(0.2)$ & $1.11(0.2)$ & $1.18(0.2)$ & $-0.4(-1.1,0.04)^{\mathrm{b}}$ & $0.292^{\mathrm{d}}$ \\
\hline TUG, s, mean (SD) & $8.2(1.5)$ & $8.5(1.5)$ & $7.8(1.5)$ & $0.5(-0.1,1.0)^{\mathrm{b}}$ & $0.075^{\mathrm{d}}$ \\
\hline \multicolumn{6}{|l|}{ Balance } \\
\hline OLS-EO, s median (IQR) & $24.7(20.2)$ & $24.7(20.6)$ & $26.5(19.8)$ & & \\
\hline Min-max & $2.2-30.0$ & $3.0-30.0$ & $2.2-30.0$ & & \\
\hline $30 \mathrm{~s}, n(\%)$ & $49(46.7)$ & $25(43.9)$ & $24(50)$ & $1.1(0.5,2.6)^{\mathrm{a}}$ & $0.839^{c}$ \\
\hline OLS-EC, median (IQR) & $3.0(3.4)$ & $3.0(3.6)$ & $3.0(3.3)$ & & \\
\hline Min-max & $0.7-30.0$ & $0.7-30.0$ & $1.1-14.5$ & & \\
\hline$<2 \mathrm{~s}, n(\%)$ & $78(74.3)$ & $43(75.4)$ & $35(72.9)$ & $1.6(0.6,4.1)^{\mathrm{a}}$ & $0.346^{\mathrm{c}}$ \\
\hline \multicolumn{6}{|l|}{ Mini-BESTest (0-28) } \\
\hline Median (IQR) & $24.0(4.0)$ & $25.0(4)$ & $23.6(3.2)$ & $1.4(0.4,2.3)^{\mathrm{b}}$ & $0.009^{\mathrm{d}}$ \\
\hline Min-max & $14-28$ & $15-28$ & $14-28$ & & \\
\hline \multicolumn{6}{|l|}{ Muscle strength } \\
\hline 30sSTS, mean (SD) & $16.9(5.1)$ & $17.6(5.1)$ & $16.2(5.0)$ & $2.1(0.3,4.0)^{\mathrm{b}}$ & $0.026^{\mathrm{d}}$ \\
\hline \multicolumn{6}{|l|}{ Grip strength, kg, mean (SD) } \\
\hline Dominant hand & $32.7(9.5)$ & $39.1(7.6)$ & $25.1(4.6)$ & $15.1(12.8,17.3)^{\mathrm{b}}$ & $<0.001^{\mathrm{d}}$ \\
\hline Non-dominant hand & $32.0(9.7)$ & $38.9(6.9)$ & $23.7(4.8)$ & $16.0(13.7,18.2)^{\mathrm{b}}$ & $<0.001^{\mathrm{d}}$ \\
\hline \multicolumn{6}{|l|}{ Endurance } \\
\hline Stair test, s, median (IQR) & $51.3(16.1)$ & $50.8(14.7)$ & $51.5(20.6)$ & $-9.3(1.7,2.9)^{\mathrm{b}}$ & $0.002^{\mathrm{d}}$ \\
\hline Min-max & $33.9-125.9$ & $33.9-103.1$ & $37.9-125.9$ & & \\
\hline
\end{tabular}

$O R$ odds ratio, $S P P B$ short physical performance battery, $O L S-E O$ one leg standing-eyes open, $O L S$-EC one leg standing-eyes closed, $30 s S T S$ 30-seconds sit-to-stand test, Mini-BESTest mini balance evaluations systems test, $T U G$ timed up and go

${ }^{\mathrm{a}} \mathrm{OR}$

${ }^{\mathrm{b}}$ Unstandardized coefficient

${ }^{c}$ Logistic regression, using dichotomized variable as dependent variable, with age as covariate

${ }^{\mathrm{d}}$ Linear regression analysis with age as covariate

Publisher's Note Springer Nature remains neutral with regard to jurisdictional claims in published maps and institutional affiliations. 